\title{
TWO-BRIDGE KNOTS WITH UNKNOTTING NUMBER ONE
}

\author{
TAIZO KANENOBU AND HITOSHI MURAKAMI
}

\begin{abstract}
We determine all two-bridge knots with unknotting number one. In fact we prove that a two-bridge knot has unknotting number one iff there exist positive integers $p, m$, and $n$ such that $(m, n)=1$ and $2 m n=p \pm$ 1 , and it is equivalent to $S\left(p, 2 n^{2}\right)$ in Schubert's notation. It is also shown that it can be expressed as $C\left(a, a_{1}, a_{2}, \ldots, a_{k}, \pm 2,-a_{k}, \ldots,-a_{2},-a_{1}\right)$ using Conway's notation.
\end{abstract}

Let $K$ be a knot in a 3-sphere. An unknotting operation is an operation which changes the overcrossing and the undercrossing at a double point of a diagram of $K$. The unknotting number of $K$, denoted by $u(K)$, is the minimum number of unknotting operations needed to deform a diagram of $K$ into that of the trivial knot, where the minimum is taken over all diagrams of $K$.

By a two-bridge knot $S(p, q)$ we mean a knot which is characterized so that its double branched covering space is the lens space $L(p, q)$, where $p$ and $q$ are coprime integers and $p$ is odd and positive $[3,6,11,12]$. (Thus we regard $S(p, q)$ and its mirror image $S(p,-q)$ as equivalent.) Let $C\left(c_{1}, c_{2}, \ldots, c_{r}\right)$ be Conway's notation for a two-bridge knot. If the continued fraction

$$
c_{1}+\frac{1}{c_{2}+\cdots} \frac{1}{+c_{r}}
$$

is equal to $p / q$, then $C\left(c_{1}, c_{2}, \ldots, c_{r}\right)$ is equivalent to $S(p, q)[\mathbf{3}, \mathbf{1 2}]$.

In this paper we consider two-bridge knots with unknotting number one and determine them. In fact we prove

THEOREM 1. Let $K$ be a nontrivial two-bridge knot. Then the following three conditions are equivalent.

(i) $u(K)=1$.

(ii) There exist an odd integer $p(>1)$ and coprime, positive integers $m$ and $n$ with $2 m n=p \pm 1$ and $K$ is equivalent to $S\left(p, 2 n^{2}\right)$.

(iii) $K$ can be expressed as $C\left(a, a_{1}, a_{2}, \ldots, a_{k}, \pm 2,-a_{k}, \ldots,-a_{2},-a_{1}\right)$.

To prove the above theorem we use the following theorem due to M. Culler, C. McA. Gordon, J. Luecke, and P. B. Shalen [4, 5] (see also Theorem A in [13]).

THEOREM 2 [5]. For a knot $K$, let $K(a / b)$ be a 3-manifold obtained by $(a / b)$ Dehn surgery along $K$, where $a$ and $b$ are coprime integers. If $K$ is not a torus knot and $\pi_{1}(K(a / b))$ is cyclic, then $|b| \leq 1$.

ProOF OF THEOREM 1. (i) $\Rightarrow$ (ii). It is known that if a nontrivial knot $K$ has unknotting number one then its double branched covering space is $\bar{K}(p / \pm 2)$

Received by the editors May 17, 1985.

1980 Mathematics Subject Classification. Primary 57M25.

Key words and phrases. Two-bridge knot, unknotting number, lens space, Dehn surgery. 
$a_{1}$ left-hand half twists $2 \varepsilon$

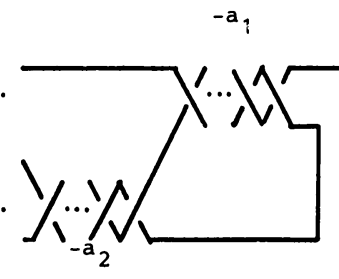

$k$ : even

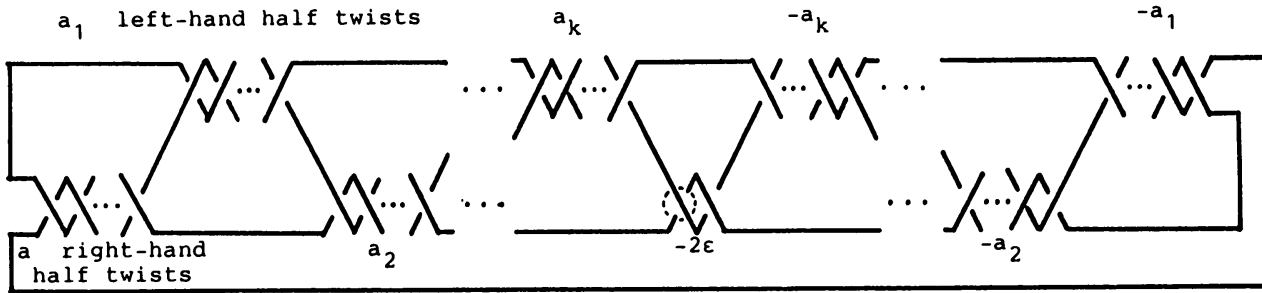

$k$ : odd

FIGURE 1

for some (strongly invertible) knot $\bar{K}$ and some positive, odd integer $p$ (see [7, 8]). Since the double branched covering space of $S(p, q)$ is the lens space $L(p, q)$, if $u(S(p, q))=1$ then $L(p, q)$ is obtainable as $\bar{k}(p / \pm 2)$ for some knot $\bar{k}$. Since $\pi_{1}(L(p, q))=\mathbf{Z} / p \mathbf{Z}, \bar{k}$ must be a torus knot from Theorem 2 .

Suppose that $\bar{k}$ is the $(m, n)$-torus $\operatorname{knot}((m, n)=1)$. From $[9]$ if $\bar{k}(p / \pm 2)$ is a lens space, $| \pm 2 m n+p|=1$ and it is homeomorphic to $L\left(p, 2 n^{2}\right)$. Thus (ii) follows.

(ii) $\Rightarrow$ (iii). First suppose that $n=1$. Then since $S(p, 2)$ is equivalent to $C((p-1) / 2,2), K$ has the required expression.

Next suppose that $n>1$. Choose integers $a(\neq 0)$ and $t$ so that $a n+t=m$ and $n>|t|>0$. Note that $(n, t)=(n, m)=1$. Then express $n / t$ by a continued fraction

Now consider continued fractions

$$
\frac{n}{t}=a_{1}+\frac{1}{a_{2}+\ldots} \frac{1}{+a_{k}}
$$

$$
f_{1}=a+\frac{1}{a_{1}+} \ldots \frac{1}{+a_{k}}+\frac{1}{2 \varepsilon}+\frac{1}{\left(-a_{k}\right)+\cdots} \frac{1}{+\left(-a_{1}\right)} \text { if } k \text { is even }
$$

and

$$
f_{2}=a+\frac{1}{a_{1}+\cdots} \frac{1}{+a_{k}}+\frac{1}{(-2 \varepsilon)}+\frac{1}{\left(-a_{k}\right)}+\cdots \frac{1}{+\left(-a_{1}\right)} \text { if } k \text { is odd }
$$

where $\varepsilon$ is chosen so that $2 m n=p-\varepsilon$.

It can be shown (using techniques described in [12] for example) that $f_{1}=f_{2}=$ $\{1+2 \varepsilon n(a n+t)\} / 2 \varepsilon n^{2}=p / 2 n^{2}$. Thus $S\left(p, 2 n^{2}\right)$ is equivalent to $C\left(a, a_{1}, a_{2}, \ldots, a_{k}\right.$, $\left.\pm 2,-a_{k}, \ldots,-a_{2},-a_{1}\right)$ in either case. (iii) follows.

(iii) $\Rightarrow$ (i). See Figure 1. The unknotting operation indicated there shows that $u(K)=1$. 


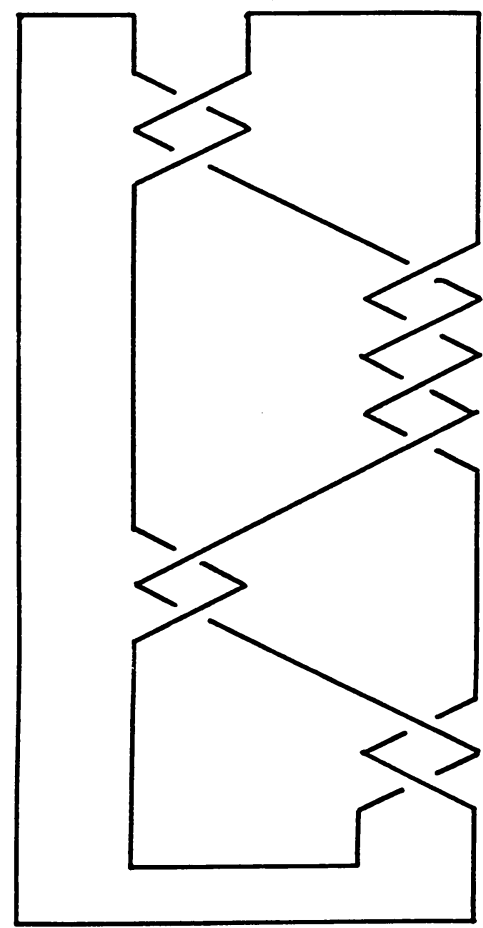

FIGURE 2

COROllary 3. $u\left(8_{3}\right)=2$. Here we use the notation of J.W. Alexander and G. B. Briggs [1].

PROOF. $8_{3}$ is the two-bridge knot $S(17,4)$. First note that $u\left(8_{3}\right) \leq 2$, as easily seen from its diagram. Suppose that $u\left(8_{3}\right)=1$. Since $(17 \pm 1) / 2=8\left(=2^{3}\right)$ or 9 $\left(=3^{2}\right), S(17,4)$ must be equivalent to $S(17,2)$ or $S\left(17,2 \times 8^{2}\right)=S\left(17,2 \times 9^{2}\right)=$ $S(17,9)$. But this is a contradiction since $S(p, q)$ is equivalent to $S\left(p, q^{\prime}\right)$ if and only if $\pm q \equiv q^{\prime}$ or $\pm q q^{\prime} \equiv 1(\bmod p)[11]$. Thus $u\left(8_{3}\right)=2$.

Using the same argument, we can conclude that the unknotting numbers of $8_{4}, 8_{6}, 8_{8}, 8_{12}, 9_{5}, 9_{8}, 9_{15}, 9_{17}$, and $9_{31}$ are all equal to two. (See [10] for a table of the unknotting numbers of knots with at most nine crossings. Another method to determine the unknotting numbers can be found in [7].)

The unknotting numbers of $8_{10}, 8_{16}, 9_{25}, 9_{32}, 9_{10}, 9_{13}, 9_{35}, 9_{38}$, and $9_{49}$ are still unknown (at least to the authors). The first four knots have unknotting numbers one or two, and the last five have unknotting numbers two or three.

Finally we give a counterexample to S. A. Bleiler's conjecture. In [2] he conjectured that the unknotting number of a two-bridge knot is realized in the diagram corresponding to a continued fraction with all coefficients even.

Let us consider the knot $8_{14}$. It is the two-bridge knot $S(31,18)$ and has unknotting number one since $31=2 \times 3 \times 5+1$ and $18=2 \times 3^{2}$. But one can show that any unknotting operation on its diagram corresponding to $C(2,-4,2,2)$ cannot create the unknot (see Figure 2). 


\section{REFERENCES}

1. J. W. Alexander and G. B. Briggs, On types of knotted curves, Ann. of Math. 28 (1927), $562-586$.

2. S. A. Bleiler, A note on unknotting number, Math. Proc. Cambridge Philos. Soc. 96 (1984), 469-471.

3. J. H. Conway, An enumeration of knots and links, and some of their algebraic properties, Computational Problems in Abstract Algebra, Pergamon Press, Oxford and New York, 1969, pp. 329-358.

4. M. Culler and P. B. Shalen, Incompressible surfaces and surgery on knots, preprint.

5. M. Culler, C. McA. Gordon, J. Luecke, and P. B. Shalen, Dehn surgery on knots, preprint, MSRI, 1985.

6. C. D. Hodgson, Involutions and isotopies of lens spaces, Master Thesis, Univ. of Melbourne, 1981.

7. W. B. R. Lickorish, The unknotting number of a classical knot, preprint, Cambridge, 1982.

8. J. M. Montesinos, Surgery on links and double branched coverings of $S^{3}$, Ann. of Math. Studies, no. 84, Princeton Univ. Press, Princeton, N. J., 1975, pp. 227-259.

9. L. Moser, Elementary surgery along a torus knot, Pacific J. Math. 38 (1971), 737-745.

10. Y. Nakanishi, A note on unknotting number, Math. Sem. Notes Kobe Univ. 9 (1981), 99-108.

11. H. Schubert, Knoten mit zwei Brücken, Math. Z. 65 (1956), 133-170.

12. L. Siebenmann, Exercices sur les nœuds rationnels, preprint, Orsay, 1975.

13. W. Whitten, Knot complements and groups, Topology (to appear).

Department of MAThematics, KyUShU UNIVERSity, HigaShi-KU, FUKUOKA, 812, JAPAN

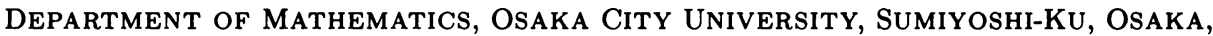
558 , JAPAN 